\title{
Macrophage Activation and Cytokine Release Syndrome in COVID-19: Current Updates and Analysis of Repurposed and Investigational Anti-Cytokine Drugs
}

\author{
Authors \\ Ashif Iqubal, Farazul Hoda, Abul Kalam Najmi, Syed Ehtaishamul Haque
}

\begin{abstract}
Affiliation
Department of Pharmacology, School of Pharmaceutical Education and Research, Jamia Hamdard, New Delhi

Key words

Cytokines, anti-inflammatory drugs, pulmonary \& respiratory pharmacology, antiviral drugs, infectious diseases
\end{abstract}

received $\quad 19.08 .2020$

accepted $\quad 13.10 .2020$

published online 12.01 .2021

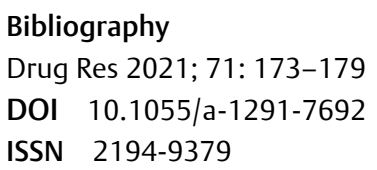

\begin{abstract}
Coronavirus disease (COVID-19) emerged from Wuhan, has now become pandemic and the mortality rate is growing exponentially. Clinical complication and fatality rate is much higher for patients having co-morbid issues. Compromised immune response and hyper inflammation is hall mark of pathogenesis and major cause of mortality. Cytokine release syndrome (CRS) or cytokine storm is a term used to affiliate the situation of hyper inflammation and therefore use of anti-cytokine and anti-inflammatory drugs is used to take care of this situation. Looking into the clinical benefit of these anti-inflammatory drugs, many of them enter into clinical trials. However, understanding the immunopathology of COVID-19 is important otherwise, indiscriminate use of these drugs could be fetal as there exists a very fine line of difference between viral clearing cytokines and inflammatory cytokines. If any drug suppresses the viral clearing cytokines, it will worsen the situation and hence, the use of these drugs must be based on the clinical condition, viral load, co-existing disease condition and severity of the infection.
\end{abstract}

\section{Introduction}

Novel coronavirus disease (COVID-19), originated from the Wuhan city of China has now taken the shape of a pandemic with a total reported case of 37.10 million and deaths of more than 1.07 million across 233 nations [1]. United State of America has a total 7.58 million confirmed cases, followed by India (7.05 million), Brazil (5.05 million), and Russia (1.29 million) as on 10/12/2020 [1]. As per the WHO report, in the USA, Brazil, and Russia, community transmission is confirmed whereas in India, yet community transmission is not reported [1]. Genomic and structural analysis of $\mathrm{nCoV}-19$ has revealed the presence of spike protein, membrane protein, envelope and nucleocapsid with the presence of 10 ORFs (operative response filament) [2]. It was found that the spike pro- tein of the virus interacts with the ACE-2 receptor located at the surface of epithelial cells and alveoli of lungs and enters into the cell via endocytosis [2]. Upon its entry, open reading frames (ORFs), nsp 1-16 and other polyproteins carryout RNA and genomic transcription and further get modified by interacting with endoplasmic reticulum and Golgi bodies. Recent findings have also confirmed the presence of nCoV-19 in the intestine, liver, and in the heart $[3,4]$. Common symptoms for COVID-19 infection are cough, fever and pneumonia leading to acute respiratory distress syndrome (ARDs) [5]. In COVID-19 infection, innate immunity plays a vital role in viral clearance. Serological analysis of COVID-19 infected patients has shown increased level of pro-inflammatory cytokines, 
which at first instance seems favorable [6]. However, in most of the critical cases, a very high level of pro-inflammatory cytokines have been reported that cause cytokine storm, which is undoubtedly a condition to worry [7]. Therefore, in this manuscript, we have critically analyzed the viral clearance and hyperinflammatory role of cytokines and therapeutic promises of anti-cytokine drugs. Further, we have discussed various mechanism of hyper inflammation that triggers ARDs towards multi-organ damage. At the end we have reviewed some of the repurposed and investigational anti-cytokine therapies in terms of their safety and efficacy. A report published in Lancet, have clarified the presence of tumor necrosis factor alpha (TNF- $\alpha$ ), interleukin (IL) IL-6, IL-10, granulocyte colonystimulating factor (G-CSF), granulocyte-macrophage colony-stimulating factor (GM-CSF), MC1, macrophage inflammatory protein 1 alpha (MIPI- $\alpha$ ) and IPI0 along with IL-17 during COVID-19 infection [5]. According to Pedersen et al., 2020, production of inflammatory cytokines is an adaptive response towards viral clearance but it was found that not all cytokines participate in viral clearance [8]. IL-12, IL-15, IL-21 and interferons (INF $\alpha, \beta$ and Y) are specific for clearance whereas an increased level of TNF- $\alpha$, IL-6, IL-17 G-CSF and GM-CSF leads to hyperinflammation and cytokine storm as shown in > Fig. 1, [9].

\section{MacrophageActivation, NLRP3Inflammasome- NF-kB mediated Hyperinflammation and Multi-organ Toxicity}

As $n C o V-19$ enters into the pneumocytes, some viral particles are engulfed by the macrophage, which serves as an initial line of defense followed by the establishment of antigen-presenting cells (APC) via major histocompatibility complex-2 (MHC-2) of T-cells [9]. This event is followed by the co-stimulation and activation of T-helper cells (CD4 ${ }^{+}$and CD8 ${ }^{+}$cells) which in turn produces IL-4 and 5 and causes activation of B-cells [9]. Antigen-presenting cells (APCs), co-stimulation, and produced cytokines also activate natural killer (NK) cells [10]. Therefore, antibodies produced from $B$ cells, granulozymes from NK cells, IL-12, 15, 21 and INF- $\alpha, \beta$ and $\gamma$ effectively participate in viral clearance $[11,12]$. However, another aspect (hyper inflammation) is mediated by macrophage activation, PMN cells, $\mathrm{CD}^{+}, \mathrm{CD} 8^{+}$cells and other signaling pathways that produce inflammatory cytokines that lead to cytokine storm and CRS (cytokine release syndrome) [13]. IL-17, produced via macrophage and polymorphonuclear neutrophils (PMNs) causes recruitment of monocytes and neutrophil leading to production and activation of downstream chemokines and cytokines such as IL-6, IL-8, and TNF- $\alpha$ and MCP-1 [14]. Thus, there are substantial evidence available that shows the overlapping function of immunopathology in COVID-19 infection and therefore the choice of anticytokine therapy must be made with caution.

Apart from the immunomodulatory effect of nCoV-2, ORFs of viral particles have been identified that interact with Toll-like receptor (TLRs) 3,7, 8 and 9 through retinoic-acid inducible gene (RIG-I), cytosolic receptor melanoma differentiation-associated gene 5 (MDA5) and nucleotidyl transferase cyclic GMP-AMP synthase (cGAS) [3,6]. This interaction recruits MAVS (mitochondrial antiviral signaling protein) and MyD88 (myeloid differentiation pri- mary response protein) that independently activate c-Jun N-terminal kinase (JNK) and nuclear factor kappa-light-chain-enhancer of activated B cells (NF-kB), respectively [15]. Activated JNK further activates $\mathrm{p} 53$ which causes apoptosis whereas activated NF-kB causes transcription of pro-inflammatory cytokines such as TNF- $\alpha$ and ILs [16, 17]. Siu et al. in 2020 showed the formation of NOD-, LRR- and pyrin domain-containing protein 3 (NLRP-3) inflammasome complex and increase in the expression of calcium channels on the pneumocytic surface [18]. NLRP3 inflammasome complex causes activation of pro-IL-1 $\beta$ whereas calcium channels increase the influx of calcium into the cells that further induces severe oxidative stress, mitochondrial dysfunction and produces inflammatory cytokines [19]. These cytokines, specifically, TNF- $\alpha$ and IL-6 are released into the systemic circulation, increases vascular permeability in the lungs and damage vital organs [20]. Considerable evidence of various organ damage such as heart, liver and kidney in COVID-19 have been reported in many case studies, supported by biopsy report and serological findings [21-23].

\section{Current Available Cytokine-based Interventions}

\section{Type-I interferon}

SARS-CoV and MERS CoV infections led to a discovery that IFN- $\alpha$ and IFN- $\beta$ can be a potential drug target for SARS-CoV-2 infection treatment [24]. Zhou et al., 2020 studied 77 patients with confirmed COVID-19 and were given either nebulized IFN- $\alpha 2 b$ ( $5 \mathrm{mU}$ b.i.d.), oral Umifenovir (200 mg t.i.d.), or a combination of both [25]. They concluded that INF- $\alpha 2 b$ has significant action in reducing the elevated inflammatory markers [25]. Another study by Wang et al., 2020 evaluated IFN- $\alpha 1 b$ in the form of nasal drops as a prophylactic measure for the healthcare staff working within the hospital [26]. A total of 2944 volunteers were included which were divided into low-risk and high-risk groups according to the exposure to coronavirus [26]. Outcome of the study showed a significant protective effect of nasal drop and showed no serious adverse effect [26]

\section{Interleukin-7}

Cytokine storm with lymphopenia and lymphocyte exhaustion are the hallmark of COVID-19 and considered to be responsible for the accumulation of viruses and reduced clearance of infected cells [11]. IL-7 is a major cytokine that is responsible for the reversal of T-cell exhaustion and promotes lymphocyte expansion, thus it becomes alluring that IL-7 may have a major role in the restoration of the immune system [27]. IL-7 also help in the replenishment of the circulation pool (CD4 + \& CD8+) of T cells by inducing potent proliferation of naïve and memory $T$ cells and also exerts anti-apoptotic activity [27]. Recombinant IL-7 (rIL-7) rIL-7 when used to treat T-cell exhaustion following septic shock in HIV patient, it was found to be therapeutically beneficial and it restored CD4 + T cell with no evidence of hyperinflammatory response [28]. Thus, use of IL-7 appears to be future therapeutics for the treatment and management of COVID-19 but as of now, there are no registered trials for evaluation of this strategy. 


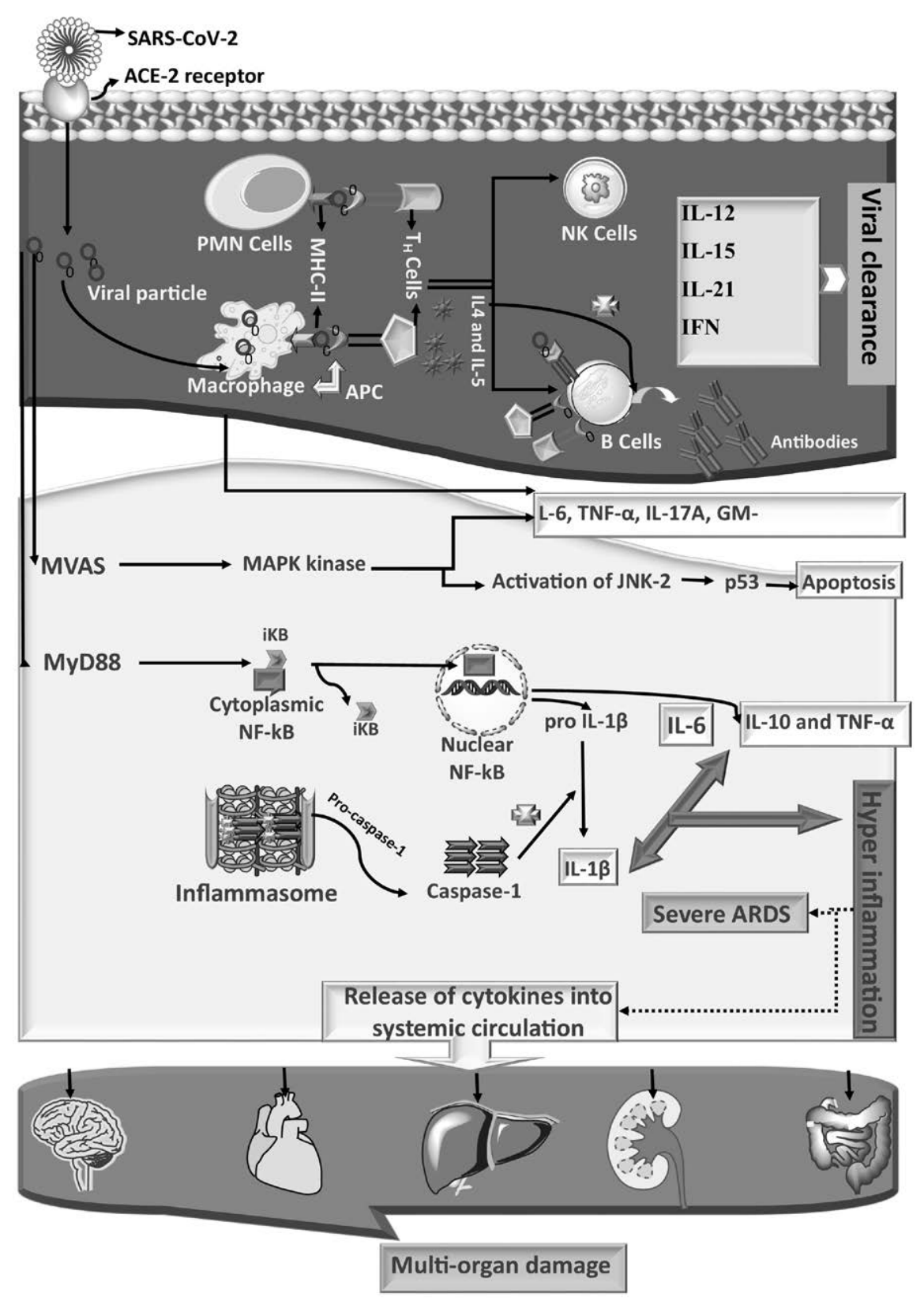

Fig. 1 Showing immunopathology of SARS-CoV-2. Upper panel showing the mechanism of viral clearance. Engulfed particles are presented at MHC-II and also identified by PMN cells leading to the formation of APC (antigen-presenting cells) which further interact with TH cells (T helper cells) and causes co-stimulation. Co-stimulated TH cells in turn produce IL- 4 and 5 that further activate B cells to produce antibodies. Activated TH cells also produce NK cells (natural killer cells) and together participate in the viral clearance. Middle panel shows the mechanism of hyper inflammation and cytokine storm. Viral particle causes recruitment of MVAS and MyD88 that causes increased activity of MAPK kinase and nuclear translocation of NF-kB leading to production p53, IL-6, TNF- $\alpha$ and pro IL- $1 \beta$. Viral particles also cause the formation of NLRP3 inflammasome that activate pro-IL- $1 \beta$ to IL-1 $\beta$. These events cumulatively cause cytokine storm and severe ARDs (acute respiratory distress syndrome) and in due course of time, these pro-inflammatory cytokines are released into the systemic circulation and cause multiorgan damage, as depicted in the lower panel.

\section{Current Available Anti-cytokine Interventions}

\section{Interleukin-6 inhibition}

IL-6 is a major mediator of cytokine storm and its serum levels have been closely correlated with the severity of ARDs and the outcome with SARS-CoV-2 viral load [20]. In a meta-analysis, 2.9 times high- er serum levels of IL-6 have been reported in patients who had complicated COVID-19 as compared to patients with non-complicated one [29]. Therefore, inhibition of IL-6 or its receptors antagonism has been found effective in the treatment of cytokine storm associated with COVID-19 complications [30]. IL-6 inhibitors include Siltuximab, Clazakizumab, Sirukumab while it's receptor inhibitors 
include Tocilizumab and Sarilumab [31]. These are approved drugs in different clinical conditions [31]. A study by Luo et al., 2020 reported increased level of IL-6 in patients with aggravated or fatal outcomes [32]. This study included 21 patients from China where 8 patients received methylprednisolone, 3 patients died while 2 experienced worsenings of the disease, 1 patient improved while 9 of them achieved clinical stability [32]. Xu et. al., 2020 reported the therapeutic effect of Tocilizumab on 21 severe or critical patients of COVID-19 [33]. This study didn't involve any control group and outcome showed a decrease in oxygen requirements (75\%), improvement in CT scan anomalies (90.5\%) and overall clinical improvement (100\%) with no deaths or adverse effects [33]. A study conducted in Italy reported clinical improvement in $33 \%$ of patients with Siltuximab at doses ranging from 700 to $1200 \mathrm{mg}$ [34]. Roumier et al., 2020 reported the outcome of Tocilizumab in 30 patients with severe pneumonia and Tocilizumab significantly reduced the requirement of ventilator and risk of ICU admission [35]. Thus, looking into the benefit of IL-6 inhibitor in COVID-19, recently, National Health Commission of China's COVID-19 diagnosis and treatment program (7th edition) included Tocilizumab in patients with extensive bilateral lung lesions opacity whereas the Infectious Diseases Society of America (IDSA), recently, published a guideline to use Tocilizumab in context with clinical trials only [36].

\section{Interleukin-1 family: IL-1 and IL-18 blockade}

A set of studies on patients with COVID-19 induced pneumonia lead to the finding that IL-1 $\beta$ and its natural antagonist (i. e. IL-1Ra) is present in the peripheral blood and bronchoalveolar lavage fluid (BALF) [37, 38]. Further, it was found that NLRP3, an inflammasome, which has been most extensively studied in MERS-CoV and SARS-CoV infection, is found to be responsible for activation and secretion of IL-1 [39]. A recombinant IL-1Ra, Anakinra, has proved it's efficacy in treating Hemophagocytic lymphohistiocytosis and it is currently used in the pharmacotherapy of IL-1 $\beta$ induced autoinflammatory disease $[40,41]$. Anakinra is being evaluated for its efficacy for COVID-19 in a clinical trial (NCT03332225). Further, a monoclonal antibody, Canakinumab, is being investigated for its action on IL-1 $\beta$ in a single-arm observational study (NCT04348448) [31]. Another upregulated cytokine, IL-18, has been observed in BALF of COVID-19 patients and mainly produced by macrophages upon the action of inflammasome-activated caspase-1 [39]. Recombinant IL-18 binding protein, Tadekinig Alfa, has shown its efficacy for the abovementioned conditions but as of now, there are no registered clinical trials to analyze its efficacy [42].

\section{Tumor Necrosis Factor- $\alpha$ inhibition}

Blood and tissue of COVID-19 patients have shown evidence of higher levels of TNF- $\alpha$ in patients with severe ARDs [43]. TNF- $\alpha$ inhibitors are well-evaluated for their efficacy in COVID-19 [44]. Neutralization of TNF- $\alpha$ in rat models showed protection against SARSCoV infection and a rapid decrease in IL- 6 and IL- 1 is observed using anti-TNF in rheumatoid arthritis patients $[45,46]$. TNF- $\alpha$ inhibitor is also responsible for the reduction of adhesion molecules and vascular endothelial growth factor, affecting capillary leak [47]. Therefore, TNF- $\alpha$ inhibitors have the potential to be a future therapeutic against COVID-19 infection.

\section{kinase inhibitors}

To take care of COVID-19 situation, various anti-inflammatory class of drugs are under trial and among them kinase inhibitors have emerged as one of the potential therapeutic modality to manage and treat COVID-19 infection [48]. These kinase inhibitors have shown 'one-drug-multiple target' properties and act on multiple proteins of the COVID-19 virus and hence, inhibit the viral entry, replication as well as mitigate the cascade of the cytokine storm [48]. As most of the cytokine inhibitors are approved for other indications, hence possess established safety and pharmacokinetic profile and therefore appears to be potential drug candidate for repurposing. Thus, looking into these facts, kinase inhibitors, if found potent in mitigating COVID-19 induced complications would significantly minimize the time as well as the cost of drug development and can be accessible to the patients in a short span of time, as compared to an entirely new class of drugs [48]. Recently, Bouhaddou et al., 2020 published an extensive study showing the significant involvement of kinases in the pathogenesis of COVID-19 and thus, opened an avenue to target these kinases against COVID19 infection [49]. Bouhaddou et al., 2020 have shown the involvement of CK2 (casein kinase II), mTOR (mammalian target of rapamycin), ERK (extracellular signal-regulated kinase), p38 MAPK (mitogen-activated protein kinase), CDKs (cyclin-dependent kinase), EGFR (epidermal growth factor receptor) and ROCK (Rho-associated protein kinases) in the pathogenesis and thus, their inhibitor appears to be the potent anti-COVID-19 therapeutics [49]. As of now many of the kinase inhibitors are under the preclinical stage and many others are under clinical trials. Silmitasertib, a CK2 inhibitor is under Phase 2 trial, Gilteritinib (AXL inhibitor, FDA approved), Bencentinib (AXL inhibitor, FDA approved), (AXL inhibitor, clinical trial), ARRY-797 and Ralimetinib (p38 MAPKs inhibitor, Phase 2/3 and Phase 2), MAPK13-IN-1 and SB203580 (p38 MAPKs inhibitor, under preclinical investigation) [50].

\section{Janus kinase (JAK) inhibitors}

JAK-STAT pathway has been well-documented in the pathogenesis of COVID-19. It plays a critical role in viral entry, viral replication and in the production of various pro-inflammatory cytokines, such as ILs, TNF- $\alpha$, GM_CSF and interferon-gamma [51]. Thus, JAK inhibitors, mainly JAK1 and JAK2 have been explored for the potential antiviral and anti-inflammatory effect in COVID-19 infection [52]. Among some of the established JAK inhibitors such as Baricitinib, Lestaurtinib, Ruxolitinib, Midostaurin, Lestaurtinib, Ruxolitinib, Fedratinib, and Tofacitinib, Baricitinib (an approved drug for rheumatoid arthritis) has shown potent protection against COVID19 infection [48, 53-55]. Interestingly, Baricitinib when used at the same dose which was approved for rheumatoid arthritis, showed inhibition of AAK1 (P2-associated protein kinase-1) and GAK (cyclin G-associated kinase) which resulted into inhibition of viral entry via ACE-2 receptors and showed potent anti-inflammatory effect leading to reduction in cytokine storm [55]. Thus, considering the therapeutic promise of Baricitinib, a Phase 2 clinical trial among 800 patients is going on where $2 \mathrm{mg}$ of Baricitinib for 10 days is being assessed for clinical safety and efficacy among COVID-19 infected patients (NCT04321993) [56]. Another Phase 2/3 clinical trial is going on where (NCT04320277) Baricitinib at the dose of $4 \mathrm{mg} /$ day in combination with Lopinavir or Ritonavir at the dose of 
$250 \mathrm{mg} /$ bid for 14 days is being evaluated for clinical efficacy [56]. Recently, Adaptive COVID-19 Treatment Trial 2 (ACTT-2) (Phase 3), where a combination of Baricitinib and Remdesivir were used, showed a significant reduction in recovery time among COVID-19 hospitalized patients [NCT04401579]. Thus, it can be concluded that JAK-inhibitors could be a potential drug to treat COVID-19 infection via its multifactorial mechanism of action.

\section{EGFR and CDKs inhibitors}

As it is well documented that pulmonary fibrosis is the clinical manifestation of COVID-19 infection and recently published evidence has shown significant pulmonary fibrosis among post-recovery patients [57], EGFR signaling is considered as the major contributor to fibrosis via modulation of the TGF- $\beta$ signaling pathway [58]. Further, TGF- $\beta$ is among the commonly reported pro-inflammatory cytokines among COVID-19 infected patients that aggravate lungs injury and ARDs. Sorafenib, a potent kinase inhibitor, as well as Osimertinib, an EGFR inhibitor, have shown significant anti-inflammatory and anti-fibrotic effect hence became a future therapeutics against COVID-19 infection [59]. Apart from EGFR inhibitor, CDK inhibitor i.e, Abemaciclib (an approved drug for advanced breast cancers) showed a significant anti-COVID-19 property and enlisted among the 24 FDA-approved drugs that possess potent inhibitory activity against the COVID-19 virus $[48,60]$.

\section{MEK inhibitors}

MEK/ERK has been reported to play a significant role in the pathogenesis of COVID-19 infection [61]. Viral spike protein and nucleocapsid proteins have been reported to stimulate the phosphorylation of ERK/MEK that triggers the release of various pro-inflammatory cytokines leading to ARDs and cytokine storm [62]. ATR-002 is a small molecule and inhibitor of Raf/MEK/ERK pathway [63]. Apart from the preclinical evidences, the outcome of the Phase I clinical trial showed the safety and clinical efficacy of ATR002 in terms of reduced virulence and cytokine storm (NCT04385420) [63]. Further, In Vitro study using ACE2-A549 has shown p38 MAPKs activation in response to COVID-19 infection and blockage of p38 MAPK pathway by its inhibitor 'SB203580' showed a significant reduction in the level of IL-6, TNF- $\alpha$ and other inflammatory cytokines [49]. Apart from SB203580, ARRY-797 and Ralimetinib (p38 MAPKs inhibitor, Phase 2/3 and Phase 2), MAPK13IN-1 (p38 MAPKs inhibitor, preclinical) are also potential MAPK inhibitors and could be a future therapeutics against COVID-19 infection [49].

\section{Analysis of Repurposed and Investigational Anti-cytokine Drugs}

As it seems very persuasive that cytokine storm is a major factor in worsening the clinical outcome of COVID-19 infection and therefore use of anti-cytokine therapy seems to be a better option. However, it must be kept in mind that there still exists a fine line of difference between viral clearance mechanism and hyper inflammation and we need to specifically stimulate the viral clearance pathway and block the hyperinflammatory pathway [64]. Looking into the inflammatory role of IL- 6 and TNF- $\alpha$, anti-IL-6 (Tocilizum$a b$, Sarilumab) and anti-TNF- $\alpha$ antibodies (Infliximab, Etanercept,
Golimumab and Adalimumab) have been used and outcomes were promising $[8,65]$. At present these drugs are under clinical trial (phase I and II). JAK-STAT pathways is yet another important signaling pathway that regulates immune and inflammatory response [66]. IL-6 has been reported to interact with JAK-STAT and activate this cascade [27]. Thus, apart from using, anti-IL-6 antibodies, JAK inhibitors can also be a novel therapeutic approach. However, use of JAK 1 and 3 inhibitors have been reported to interact and inhibit IL-15, IL-21 and INFs, which is essential for viral clearance and inhibition of these cytokines could be fetal [9]. However, the use of the JAK2 inhibitor has been reported to inhibit IL-17 (responsible for cytokine activation), IL-6 and GM-CSF which are responsible for cytokine storm [67]. This seems to be a favorable approach. Apart from the aforementioned drugs, several other anti-inflammatory drugs such as NSAIDs, Sulfasalazine, Mesalazine and COX-2 inhibitors are being investigated $[3,68]$ but we strongly suggest to take care while selecting these anti-cytokine/anti-inflammatory drugs, as slightest of deviation can be fetal for patients.

\section{Conclusion and Future Direction}

Looking into the pathological role of cytokine storm and previous background of anti-cytokine activity of anti-IL-6 or TNF $\alpha$ antibody, we speculate that the use of these drugs can be beneficial but use must be on a case to case basis. We also need to think of two aspects regarding the use of these drugs (1) when anti-cytokine therapy should be given? and (2), how safe these drugs are?. Based on the published reports, it can be said that early use of anti-cytokine therapy will be a better option, as compared to steroidal therapy which is meant for immunosuppression [69]. Use of these drugs should be avoided in case, patients are in intensive care. Concerning the safety, use of high dose anti-inflammatory drugs, in general causes tissue damage and potent anti-inflammatory drugs increase the risk of supra infection (bacterial and fungus infection). However, till now, no such case of supra-infection have reported. Therefore, in nutshell, anti-cytokine drugs have the potential to be a choice of drugs for the management and treatment of COVID-19 associated ARDs and cytokine storm but large sample size study will only decide their forthcoming future. Until now, for a healthy individual, social distancing along with an active lifestyle and for critically ill patients, plasma therapy appears to be a safer alternative, unless vaccine is introduced.

\section{Conflict of Interest}

The authors declare that they have no conflict of interest.

\section{References}

[1] Disease WC: WHO COVID-19 update. 2020

[2] Munster V], Koopmans $\mathrm{M}$, van Doremalen $\mathrm{N}$ et al. A novel coronavirus emerging in China-key questions for impact assessment. New England Journal of Medicine 2020; 382: 692-694

[3] Vellingiri B, Jayaramayya K, lyer M et al. COVID-19: A promising cure for the global panic. Science of the Total Environment 2020; 138277 
[4] Shi C-S, Nabar NR, Huang N-N et al. SARS-Coronavirus Open Reading Frame-8b triggers intracellular stress pathways and activates NLRP3 inflammasomes. Cell Death Discovery 2019; 5: 1-12

[5] Wang D, Hu B, Hu C et al. Clinical characteristics of 138 hospitalized patients with 2019 novel coronavirus-infected pneumonia in Wuhan, China. 2020; 323: 1061-1069

[6] Grasselli G, Zangrillo A, Zanella A et al. Baseline Characteristics and Outcomes of 1591 Patients Infected With SARS-CoV-2 Admitted to ICUs of the Lombardy Region, Italy. Jama 2020; $1-8$

[7] Mehta P, McAuley DF, Brown M et al. COVID-19: consider Cytokine storm syndromes and immunosuppression. The Lancet 2020; 395 1033-1034

[8] Monteleone G, Sarzi-Puttini PC, Ardizzone S. Preventing COVID-19-induced pneumonia with anticytokine therapy. The Lancet Rheumatology 2020; 2: e255-e256

[9] Schett G, Sticherling M, Neurath MF. COVID-19: Risk for cytokine targeting in chronic inflammatory diseases? Nature Reviews Immunology 2020; 1-2

[10] Thomassen M], Divis LT, Fisher CJ. Regulation of human alveolar macrophage inflammatory cytokine production by interleukin-10. Clinical Immunology and Immunopathology 1996; 80: 321-324

[11] Henderson LA, Canna SW, Schulert GS et al. On the alert for cytokine storm: Immunopathology in COVID[19. Arthritis \& Rheumatology 2020; 72: 1059-1063

[12] Rossol S, Marinos G, Carucci P et al. Interleukin-12 induction of Th1 cytokines is important for viral clearance in chronic hepatitis $\mathrm{B}$. The. Journal of Clinical Investigation 1997; 99: 3025-3033

[13] Gupta L, Agarwal V, Ramanan AV: Interleukin-6 and other cytokine blockade in COVID-19 hyperinflammation. Indian J Rheumatol 2020

[14] Amatya N, Garg AV, Gaffen SL. IL-17 signaling: the yin and the yang. Trends in Immunology 2017; 38: 310-322

[15] Huang Y, Liu H, Li S et al. Wang CJPp: MAVS-MKK7-JNK2 defines a novel apoptotic signaling pathway during viral infection 2014; 101 : e1004020

[16] Fuchs SY, Adler V, Pincus MR, Ronai Ze: MEKK1/JNK signaling stabilizes and activates p53. Proceedings of the National Academy of Sciences 1998, 95

[17] lqubal A, Sharma S, Ansari MA et al. Nerolidol attenuates cyclophosphamide-induced cardiac inflammation, apoptosis and fibrosis in Swiss Albino mice. European Journal of Pharmacology 2019; 863: 172666

[18] Siu K-L, Yuen K-S, Castaño-Rodriguez C et al. Severe acute respiratory syndrome coronavirus ORF3a protein activates the NLRP3 inflammasome by promoting TRAF3-dependent ubiquitination of ASC. The FASEB Journal 2019; 33: 8865-8877

[19] Liu D, Zeng X, Li X et al. Advances in the molecular mechanisms of NLRP3 inflammasome activators and inacativators. Biochemical Pharmacology 2020; 113863

[20] Liu B, Li M, Zhou Z et al. Can we use interleukin-6 (IL-6) blockade for coronavirus disease 2019 (COVID-19)-induced cytokine release syndrome (CRS)? Journal of Autoimmunity 2020; 102452

[21] Xu Z, Shi L, Wang Y et al. Pathological findings of COVID-19 associated with acute respiratory distress syndrome. The Lancet Respiratory Medicine 2020; 8: 420-422

[22] Yao X, Li T, He Z et al. A pathological report of three COVID-19 cases by minimally invasive autopsies. Zhonghua bing li xue za zhi = Chinese Journal of Pathology 2020; 49: E009-E009

[23] Wang T, Du Z, Zhu F et al. Comorbidities and multi-organ injuries in the treatment of COVID-19. The Lancet 2020; 395: e52

[24] Park A, Iwasaki A. Type I and Type III Interferons-Induction, Signaling, Evasion, and Application to Combat COVID-19. Cell Host \& Microbe 2020; $27: 870-878$
[25] Zhou Q, Wei X-S, Xiang X et al. Interferon-a2b treatment for COVID-19. MedRxiv 2020; 11: 1061

[26] Meng Z, Wang T, Li C et al. An experimental trial of recombinant human interferon alpha nasal drops to prevent coronavirus disease 2019 in medical staff in an epidemic area. MedRxiv 2020; Preprint

[27] Nanjappa SG, Kim EH, Suresh M. Immunotherapeutic effects of IL-7 during a chronic viral infection in mice. Blood, The Journal of the American Society of Hematology 2011; 117: 5123-5132

[28] Wang YY, Attané C, Milhas D et al. Mammary adipocytes stimulate breast cancer invasion through metabolic remodeling of tumor cells. JCI Insight 2017; 2: e87489

[29] Coomes EA, Haghbayan H. Interleukin-6 in COVID-19: A systematic review and meta-analysis. MedRxiv 2020; e2141

[30] Zhang W, Zhao Y, Zhang $F$ et al. The use of anti-inflammatory drugs in the treatment of people with severe coronavirus disease 2019 (COVID-19): The experience of clinical immunologists from China. Clinical Immunology 2020:108393

[31] Jamilloux Y, Henry T, Belot A et al. Should we stimulate or suppress immune responses in COVID-19? Cytokine and anti-cytokine interventions. Autoimmunity Reviews 2020; 102567

[32] Luo P, Liu Y, Qui L et al. March 2020, posting date. Tocilizumab treatment in COVID-19: A single center experience. J Med Virol 2020; 92: 814-818 doi, 10

[33] Xu X, Han M, Li T et al. Effective treatment of severe COVID-19 patients with tocilizumab. Proceedings of the National Academy of Sciences 2020; 117: 10970-10975

[34] Gritti G, Raimondi F, Ripamonti D et al. Use of siltuximab in patients with COVID-19 pneumonia requiring ventilatory support. MedRxiv 2020; Preprint

[35] Roumier M, Paule R, Matthieu G, et al. Interleukin-6 blockade for severe COVID-19. medrxiv 2020; Preprint

[36] Bhimraj A, Morgan RL, Shumaker AH, et al. Infectious diseases Society of America guidelines on the treatment and management of patients with COVID-19. Clinical Infectious Diseases 2020; ciaa478

[37] Xiong Y, Liu Y, Cao L et al. Transcriptomic characteristics of bronchoalveolar lavage fluid and peripheral blood mononuclear cells in COVID-19 patients. Emerging Microbes \& Infections 2020; 9: 761-770

[38] Huang C, Wang Y, Li X et al. Clinical features of patients infected with 2019 novel coronavirus in Wuhan, China. The Lancet 2020; 395: 497-506

[39] Martinon F, Burns K, Tschopp J. The inflammasome: a molecular platform triggering activation of inflammatory caspases and processing of prolL- $\beta$. Molecular Cell 2002; 10: 417-426

[40] Wampler Muskardin TL. Intravenous Anakinra for Macrophage Activation Syndrome May Hold Lessons for Treatment of Cytokine Storm in the Setting of Coronavirus Disease 2019. ACR Open Rheumatology 2020; 2: 283-285

[41] Jamilloux Y, Belot A, Magnotti F et al. Geoepidemiology and immunologic features of autoinflammatory diseases: A comprehensive review. Clinical Reviews in Allergy \& Immunology 2018; 54: 454-479

[42] Weiss ES, Girard-Guyonvarc'h C, Holzinger D et al. Interleukin-18 diagnostically distinguishes and pathogenically promotes human and murine macrophage activation syndrome. Blood 2018; 131: 1442-1455

[43] Liu Y, Du X, Chen J et al. Neutrophil-to-lymphocyte ratio as an independent risk factor for mortality in hospitalized patients with COVID-19. Journal of Infection 2020; 81: e6-e12

[44] Feldmann M, Maini RN, Woody JN et al. Trials of anti-tumour necrosis factor therapy for COVID-19 are urgently needed. The Lancet 2020; 395: 1407-1409

[45] McDermott JE, Mitchell HD, Gralinski LE et al. The effect of inhibition of PP1 and TNFa signaling on pathogenesis of SARS coronavirus. BMC Systems Biology 2016; 10: 1-12 
[46] Charles P, Elliott M], Davis D et al. Regulation of cytokines, cytokine inhibitors, and acute-phase proteins following anti-TNF- $\alpha$ therapy in rheumatoid arthritis. The Journal of Immunology 1999; 163: $1521-1528$

[47] Paleolog EM, Young S, Stark AC et al. Modulation of angiogenic vascular endothelial growth factor by tumor necrosis factor $\alpha$ and interleukin-1 in rheumatoid arthritis. Arthritis \& Rheumatism 1998; 41: 1258-1265

[48] Weisberg E, Parent A, Yang PL et al. Repurposing of Kinase Inhibitors for Treatment of COVID-19. Pharmaceutical Research 2020; 37: 1-29

[49] Bouhaddou M, Memon D, Meyer B et al. The global phosphorylation landscape of SARS-CoV-2 infection. Cell 2020; 182: 712. e685-712. e619

[50] Gordon D, Jang G, Bouhaddou M et al. A SARS-CoV-2 protein interaction map reveals targets for drug repurposing. Nature 2020; 30 $-2020$

[51] Luo W, Li Y-X, Jiang L-J et al. Targeting JAK-STAT signaling to control cytokine release syndrome in COVID-19. Trends in Pharmacological Sciences 2020; 41: 531-543

[52] Bagca BG, Avci CB. Overview of the COVID-19 and JAK/STAT Pathway Inhibition: Ruxolitinib Perspective. Cytokine \& Growth Factor Reviews 2020; 54: 51-61

[53] Yazici Y, Regens AL. Promising New Treatments for Rheumatoid Arthritis. Bulletin of the NYU Hospital for Joint Diseases 2011; 69: 233-237

[54] Ярилин А: Иммунология: учебник./Ярилин А. А. Москва ГЭОТАРМедиа 2010; 752 c

[55] Richardson P, Griffin I, Tucker C et al. Baricitinib as potential treatment for 2019-nCoV acute respiratory disease. Lancet. In.: Lancet Publishing Group 2020; 395: e30-e31

[56] Seif F, Aazami H, Khoshmirsafa M et al. JAK inhibition as a new treatment strategy for patients with COVID-19. International Archives of Allergy and Immunology 2020; 181: 467-475

[57] George PM, Wells AU, Jenkins RG. Pulmonary fibrosis and COVID-19: the potential role for antifibrotic therapy. The Lancet Respiratory Medicine 2020; 8: 807-815
[58] Venkataraman T, Frieman MB. The role of epidermal growth factor receptor (EGFR) signaling in SARS coronavirus-induced pulmonary fibrosis. Antiviral Research 2017; 143: 142-150

[59] Karaś K, Sałkowska A, Karwaciak I et al. The Dichotomous Nature of AZ5104 (an EGFR Inhibitor) Towards RORy and RORYT. International Journal of Molecular Sciences 2019; 20: 5780

[60] Jeon S, Ko M, Lee J et al. Identification of antiviral drug candidates against SARS-CoV-2 from FDA-approved drugs. Antimicrobial Agents and Chemotherapy 2020; 64: e00819-e00820

[61] Bello-Perez M, Sola I, Novoa B et al. Canonical and noncanonical autophagy as potential targets for COVID-19. Cells 2020; 9: 1619

[62] Wehbe Z, Hammoud S, Soudani N et al. Molecular Insights Into SARS COV-2 Interaction With Cardiovascular Disease: Role of RAAS and MAPK Signaling. Frontiers in Pharmacology 2020; 11: 836

[63] Laure M, Hamza H, Koch-Heier J et al. Antiviral efficacy against influenza virus and pharmacokinetic analysis of a novel MEK-inhibitor, ATR-002, in cell culture and in the mouse model. Antiviral Research 2020; 104806

[64] Merad M, Martin JC. Pathological inflammation in patients with COVID-19: a key role for monocytes and macrophages. Nature Reviews Immunology 2020; 1-8

[65] Khan F, Fabbri L, Stewart I et al. A systematic review of Anakinra, Tocilizumab, Sarilumab and Siltuximab for coronavirus-related infections. medRxiv 2020; Preprint

[66] Tamiya T, Kashiwagi I, Takahashi R et al. Suppressors of cytokine signaling (SOCS) proteins and JAK/STAT pathways: Regulation of T-cell inflammation by SOCS1 and SOCS3. Arteriosclerosis, Thrombosis, and Vascular Biology 2011; 31: 980-985

[67] Wu D, Yang XO. TH17 responses in cytokine storm of COVID-19: An emerging target of JAK2 inhibitor Fedratinib. Journal of Microbiology, Immunology and Infection 2020; 53: 368-370

[68] Cascella M, Rajnik et al. Features, evaluation and treatment coronavirus (COVID-19). In: Statpearls [internet]. StatPearls Publishing; 2020

[69] Ritchie Al, Singanayagam A. Immunosuppression for hyperinflammation in COVID-19: A double-edged sword? The Lancet 2020; 395: 1111 